\title{
Opportunities to improve image quality in PET myocardial viability imaging in diabetics
}

\author{
Brett W. Sperry, $M D^{\mathrm{a}, \mathrm{b}}$ \\ a Saint Luke's Mid America Heart Institute, Kansas City, MO \\ b University of Missouri - Kansas City, Kansas City
}

Received Aug 16, 2021; accepted Aug 17, 2021

doi: $10.1007 / \mathrm{s} 12350-021-02792-7$

\section{See related article, pp. 2497-2507}

There are three ways of doing things around here: the right way, the wrong way, and the way that I do it.

—Robert DeNiro

Imaging myocardial viability in the modern era is accomplished with either cardiac magnetic resonance imaging (MRI) or 18F-fluorodeoxyglucose positron emission tomography (FDG PET). The benefit of cardiac MRI is accuracy in the detection of myocardial scar, tissue characterization, and better spatial resolution than PET. Regional wall motion, ischemia, and coronary flow reserve can also be assessed. ${ }^{1}$ The presence of MRI unsafe hardware is a contraindication to imaging, and even conditional or safe devices may lead to artifact, limiting interpretability. Chronic kidney disease is also a concern with gadolinium agents, and low dose or no contrast may be considered. FDG PET does not have these limitations, and provides quantification of myocardial metabolism, and therefore viability. Additional data from PET includes quantification of resting perfusion defects, ischemia, peak stress wall motion, and myocardial blood flow reserve.

The difficulty with FDG PET lies in the pre-procedure glucose manipulation protocol. The myocardium metabolizes $60 \%-70 \%$ of its energy through free fatty acid oxidation when in the fasting state, while glucose accounts for most of the energy utilization in the fed

Reprint requests: Brett W. Sperry, MD, Saint Luke's Mid America Heart Institute, 4401 Wornall Rd, Kansas City, MO 64111; bsperry@saintlukeskc.org

J Nucl Cardiol 2022;29:2508-10.

$1071-3581 / \$ 34.00$

Copyright (c) 2021 American Society of Nuclear Cardiology. state. $^{2}$ Recommended glucose manipulation protocols typically involve ingestion of oral glucose followed by insulin administration to simulate the fed state and drive glucose into the myocardium. Once there is a decrease in blood glucose levels from the effects of insulin, F18FDG is administered and taken up by GLUT1 and GLUT4 transporters in the myocardium. Glucose manipulation can be particularly challenging in diabetic patients, where studies have demonstrated impaired myocardial glucose transport and elevated free fatty acid levels, resulting in diminished glucose metabolism in myocardial cells. ${ }^{3}$ This can lead to difficulties in interpreting FDG PET studies in diabetics.

Over time, interventions like strict glucose manipulation protocols, the hyperinsulinemic-euglycemic clamp, and acipimox (not available in the USA) have improved glucose uptake and image quality in diabetic patients. ${ }^{4}$ Acipimox is a potent nicotinic acid derivative that rapidly reduces serum free fatty acid levels by inhibiting lipolysis in peripheral tissue. It has been shown to perform similarly to the insulin clamp with respect to uptake of myocardial glucose and distinction between normal, mismatch, and scar territories. ${ }^{5}$ Acetaminophen is sometimes given in order to minimize systemic vasodilatory side effect. Niacin is also a nicotinic acid derivative and is available in the USA, but its use prior to FDG PET did not result in improved image quality in diabetics when compared with oral glucose load and insulin. ${ }^{6}$

The current study by Shao et al examined trimetazidine hydrochloride (a cardiac energy metabolism regulator, not available in the USA) as pre-treatment prior to FDG PET for viability as a potential addition to the armamentarium of options to improve myocardial glucose utilization in diabetics. This medication helps to transform myocardial energy utilization from free fatty acids to glucose and was initially developed as an antianginal medication, though there are data that it may also benefit patients with heart failure and reduced 
ejection fraction. ${ }^{7}$ The hypothesis of this study was that oral administration of trimetazidine before FDG PET would improve image quality in diabetics. The authors prospectively enrolled 23 diabetic patients (12 on insulin) in 2017 who underwent rest $99 \mathrm{~m}$ technetium sestamibi perfusion imaging on day 1, a FDG PET viability study with standard oral glucose load and intravenous insulin on day 2, and the same FDG PET viability study with oral glucose load, intravenous insulin, and premedication with 2 doses of trimetazidine on day 3. FDG PET images were graded as good, moderate, or uninterpretable based upon myocardial uptake and background activity.

The results showed that trimetazidine did not alter serum glucose levels, but did improve image quality in almost half of the patients (11 of 23). Specifically, 3 of 4 uninterpretable image sets without trimetazidine became interpretable after administration, and the overall number of myocardial segments graded as viable increased. While trimetazidine increased F18-FDG uptake across the spectrum of perfusion defect severities, the largest increases were noted in segments with mild resting perfusion defects. As these segments are most likely to be hibernating/viable, it follows that a medication improving myocardial glucose utilization will have the most dramatic effect in these areas. Quantitatively, myocardial SUVs increased with trimetazidine (mean SUV $3.11+/-1.07$ versus $2.32+/$ - 1.00). Left ventricular end diastolic volume assessed on FDG PET also increased by an average of $34 \mathrm{~mL}$ with trimetazidine and was more correlated with echocardiography-derived volume. This is likely secondary to increased myocardial F18-FDG uptake leading to improved detection of myocardial borders. The authors conclude that this medication is safe and effective to improve success rate and image quality in diabetic patients with severe CAD.

The first point to consider is that, even in diabetics, FDG PET quality is fairly good with standard protocols (only $17 \%$ uninterpretable in this study). Sometimes adjusting the color scheme of F18-FDG metabolism images by normalizing counts to the myocardial region with normal perfusion or blood flow ${ }^{4}$ can make up for these issues. Second, IV dextrose (instead of oral glucose) is another option included in the guidelines, ${ }^{4}$ and anecdotally, our lab has noted improved quality of F18FDG images after routinely switching to this protocol. Intravenous dextrose has been shown to lead to shorter protocols than oral glucose with improved image quality. ${ }^{8}$ Nuclear labs will have to decide a strategy to use in diabetics a priori, and IV dextrose may be easier than the other strategies (Figure 1), particularly in the USA where acipimox and trimetazidine are not available. Additionally, a rescue protocol of reinjected low-dose 18F-FDG with simultaneous insulin and glucose can

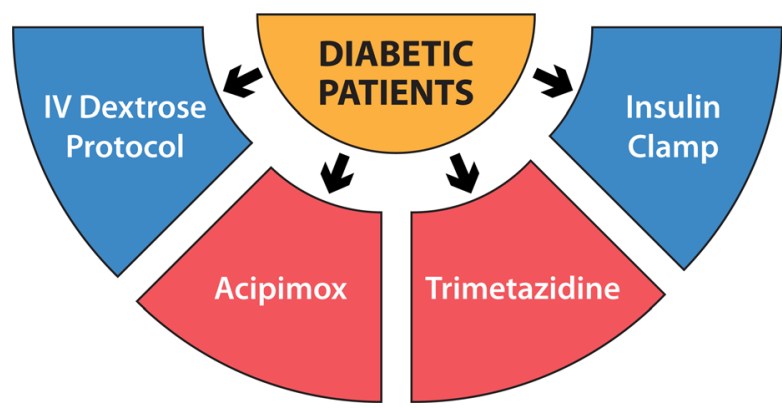

Figure 1. Options to improve image quality in FDG PET imaging. Four options have been described to improve image quality in diabetic patients undergoing FDG PET imaging for viability instead of the standard oral glucose protocol: intravenous dextrose, the insulin clamp, and pretreatment with either acipimox, or trimetazidine.

improve the image quality of FDG PET images that are of poor quality. ${ }^{9}$

There have only been 2 prior studies analyzing FDG PET images in patients taking trimetazidine, both with long term use. These studies had conflicting results, and whether or not trimetazidine affects myocardial metabolism in the long term is unclear. Future studies with trimetazidine in both the short and long term are warranted.

There are multiple techniques to improve image quality for FDG PET viability studies in patients with diabetes; there is not a "right way" or "wrong way", and it appears that trimetazidine is a viable fourth option. But for now, "the way I do it" will still be with intravenous dextrose which provides good image quality and shorter imaging protocols. Future studies should compare these four options for image optimization and correlate subsequent FDG PET findings with outcomes after revascularization.

\section{Disclosures}

No relevant disclosures.

\section{References}

1. Almeida AG, Carpenter J-P, Cameli M, Donal E, Dweck MR, Flachskampf FA, et al. Multimodality imaging of myocardial viability: An expert consensus document from the European Association of Cardiovascular Imaging (EACVI). Eur Heart $\mathbf{J}$ Cardiovasc Imaging 2021;22(8):e97-125. https://doi.org/10.1093/eh jci/jeab053

2. Neely JR, Rovetto MJ, Oram JF. Myocardial utilization of carbohydrate and lipids. Prog Cardiovasc Dis 1972;15(3):289-329. https://doi.org/10.1016/0033-0620(72)90029-1 
3. vom Dahl J, Herman WH, Hicks RJ, Ortiz-Alonso FJ, Lee KS, Allman KC, et al. Myocardial glucose uptake in patients with insulin-dependent diabetes mellitus assessed quantitatively by dynamic positron emission tomography. Circulation 1993;88(2):395-404. https://doi.org/10.1161/01.CIR.88.2.395

4. Dilsizian V, Bacharach SL, Beanlands RS, Bergmann SR, Delbeke $\mathrm{D}$, Gropler RJ, et al. ASNC imaging guidelines/SNMMI procedure standard for positron emission tomography (PET) nuclear cardiology procedures. J Nucl Cardiol 2016;23(5):1187-226. https://doi. org/10.1007/s12350-016-0522-3

5. Knuuti MJ, Yki-Järvinen H, Voipio-Pulkki LM, Mäki M, Ruotsalainen U, Härkönen $\mathrm{R}$, et al. Enhancement of myocardial [fluorine-18]fluorodeoxyglucose uptake by a nicotinic acid derivative. J Nucl Med 1994;35(6):989-98

6. Vitale GD, deKemp RA, Ruddy TD, Williams K, Beanlands RS. Myocardial glucose utilization and optimization of (18)F-FDG PET imaging in patients with non-insulin-dependent diabetes mellitus, coronary artery disease, and left ventricular dysfunction. J Nucl Med 2001;42(12):1730-6
7. Fragasso G, Palloshi A, Puccetti P, Silipigni C, Rossodivita A, Pala $\mathrm{M}$, et al. A randomized clinical trial of trimetazidine, a partial free fatty acid oxidation inhibitor, in patients with heart failure. J Am Coll Cardiol 2006;48(5):992-8. https://doi.org/10.1016/j.jacc.2006. 03.060

8. Martin WH, Jones RC, Delbeke D, Sandler MP. A simplified intravenous glucose loading protocol for fluorine-18 fluorodeoxyglucose cardiac single-photon emission tomography. Eur $\mathbf{J}$ Nucl Med 1997;24(10):1291-7. https://doi.org/10.1007/ s002590050154

9. Sun X-X, Li S, Wang Y, Li W, Wei H, He Z-X. Rescue protocol to improve the image quality of 18F-FDG PET/CT myocardial metabolic imaging. Clin Nucl Med 2021;46(5):369-74. https://doi. org/10.1097/RLU.0000000000003572

Publisher's Note Springer Nature remains neutral with regard to jurisdictional claims in published maps and institutional affiliations. 